\title{
Review of: "Risk of Plasmodium falciparum infection in south-west Burkina Faso - potential impact of expanding eligibility for seasonal malaria chemoprevention"
}

\author{
Abdunoor Mulokozi Kabanywanyi ${ }^{1}$
}

1 Ifakara Health Institute (IHI)

Potential competing interests: The author(s) declared that no potential competing interests exist.

This Manuscript has Minor and Major faults that I would recommend a halt of publication until they are fully attended.

\section{Minor:}

Study design

1. There is no mention throughout the design section on whether this was a household or health facility survey

2. In case it was a household survey how many households were involved and how each household was expected to yield enough number of subject and how was that predetermined.

3. $12 \%$ of subjects i.e., 150 study subjects (50 in each age strata) were randomly selected from the Health and Demographic Surveillance System (HDSS). It is not clear what was the purpose of this sampling and how was this used to measure what?

4. It is further indicated that 150 of participants listed from 10 study villages were entered in the screening process...What was the essence of this sampling and how this was treated in analyses. Which screening is being referred here?

5. The author indicates that Blood spots were taken for polymerase chain reaction analysis but failed to amplify any PCR results. However, there is no clarification if that was due to technique error, poor sample preparations or poor lab handling and performance. Which also should have been mentioned in the limitation. The absence of submicroscopic indices of parasites has huge parasitaemia results' validity implication.

Major: (without resolution I may not recommend it to publish) Study design

1. Despite the planned random sampling in which individuals from ten villages representatives of three age strata in ( 2 to $<10$ years, 10 to $<30$ years and $\geq 30$ years) with 400 subjects each were recruited, giving a total sample size of 1,200 individuals the analyses thereafter were contrary based on a different sampling frame of (instead) a 5 age strata. 


\section{Analysis}

2. Sample size was estimated based on a power of showing a difference in prevalence of parasitemia between 40 to $60 \%$ across three age groups. Analysis instead was moreover done based on 5 groups which was also devoid of clarification about why this was the case and when was it decided about.

3. Despite the intention to show strata based proportions of malaria indicators as characterized in the methods text section, in the analyses instead the Author adopted a scale of five groups who were not only unequal by size of individuals included in each but also by arbitrary new cut off points as opposed to what was initially thought in the design.

4. It is stated in the design that parasite prevalence was estimated as the proportion of subjects infected divided by the (total) number of subjects tested, but instead this prevalence is determined in the subgroups using some strata-based denominators which were not only confused, but also not representative of the study sampling frame.

5. The choice of denominator for various parameters in table 1 is not representative of the study and to some extent they carry no meaningful results' message. For instance, one may wonder why instead of referring parasite and other malaria measured parameters on common denominator the author opted a strata oriented approach. For instance, one would argue what was the essence of (extrapolations) reporting that: There was overall $1.7 \%$ of mixed infection in the study population and $4.9 \%$ of them was among the age group of those who were between 5 to 15 years? Because of this confusion the main current descriptive results are totally unfit to describe the study findings likewise the conclusion.

6. The Author accidentally has mentions in the discussion section that “.... used post-hoc age strata in the analysis, and further contends that the large sample size was sufficient to identify significant differences between the under 5 years old, 5-10 years old and 10-15 years old children". However, the Author fails to justify: When was the post-hoc approach decided and what was the basis and which power was the decision based on? The entire methods section is devoid of this clarification, and one finds the mention lightly in the discussion. Of course one may argue that if then this was the case the Author would probably have provided its narratives in the limitations section by elaborating, why was this a choice? It is established that a post-hoc analysis is somewhat fraught with limitations - which in some cases it is - then probably if one opts this, would have been of good service to the readers, indicating why was it an option? 